\title{
Chapter 48 \\ A Final Word: The Value of Urban \\ Informatics
}

\author{
Michael F. Goodchild
}

\subsection{Introduction}

The chapters of this book include a rich collection of novel forms of data acquisition, techniques of analysis and visualization, and broader concerns about such topics as privacy, urban governance, and urban planning. It is clear from this outpouring of material that urban informatics is a large and burgeoning field. In some cases, especially the chapters in Part IV, the objectives have been the traditional ones of science: the acquisition of new and general knowledge, in the tradition of the UK's Royal Society (to give it its full seventeenth-century title as devised by Isaac Newton and others: the Royal Society of London for Improving Natural Knowledge). In other cases, the objectives are more those of planning; they are normative, in the sense that they assume an ability to design and intervene according to certain principles, using established scientific knowledge. In yet other cases, the authors have been satisfied simply to report capabilities and to discuss the new kinds of data that urban informatics is generating, without any explicit statement of the objectives to which those capabilities and new data are to be applied or how value should be assessed. The finale of the book seems an appropriate place to indulge such broader issues of context.

Several chapters have been concerned with big data, which they have defined in terms of characteristics beginning with $\mathrm{V}$ (see, for example, Chap. 43, which cites five Vs: volume, variety, velocity, veracity, and value). Volume, variety, and velocity are central to discussions of big data: volume implying an abundance of data, variety implying a multiplicity of sources, and velocity implying near-real time. Veracity clearly refers to data quality, which big data often lack when compared to more traditional data-production programs; in a sense, then, the fourth $\mathrm{V}$ might be identified as an anti-V. Including value, however, begs the question of purpose:

M. F. Goodchild ( $\varangle)$

University of California, Santa Barbara, Santa Barbara, USA

e-mail: good@geog.ucsb.edu 
whose interests are served by big data? More fundamentally, we can ask the same question about urban informatics: whose interests does it serve, and whose interests are marginalized?

To what extent should specialists in urban informatics concern themselves with these issues? In the early 1990s, a number of scholars drew attention to the social implications of geographic information systems (GIS; Pickles 1995; Schuurman 2000), with the implicit or explicit suggestion that developers of GIS were ignoring such concerns. Much of the early technical development of GIS originated in Eisenhower's military-industrial complex, where its purposes could easily be seen as diametrically opposed to the immediate concerns of a civilian society (Smith 1992). GISs were being used even then to track and monitor citizens (https://www.co.pierce. wa.us/1964/Sex-Offenders-in-Pierce-County), and today geospatial technologies are an essential part of many programs of public surveillance (Chap. 32). Asking these questions about urban informatics recalls the kinds of soul-searching that occurred during and after the development of the atomic bomb, though that case is clearly more extreme. For example, it is hard to imagine anyone working in urban informatics to be driven, as Oppenheimer was on witnessing the first nuclear explosion, to quote from the Bhagavad Gita: "Now I am become death, the destroyer of worlds" (https://www.wired.co.uk/article/manhattan-project-robert-oppenheimer). Nevertheless, it seems appropriate at the end of the book to enquire about that fifth $\mathrm{V}$ and its implications for the future. What kind of urban world is likely to result from all of this research and development, and what can be done to ensure that the field moves in a positive rather than a negative direction? In developing and advancing urban informatics, are we headed for a future utopia, and what kinds of dystopias might emerge as unforeseen and unintended consequences? Are we, like Mark Zuckerberg and the early days of Facebook, in favor of technical disruption for its own sake (Taplin 2017), or would we rather a more considered future, a slow urban informatics if you like? In short, what constitutes value in urban informatics?

To focus this discussion of the bigger picture somewhat, the next section proposes several alternative visions of what urban informatics is about, and its corresponding form of accountability.

\subsection{Visions for Urban Informatics}

\subsubsection{Urban Intelligence}

James Clapper, who retired in 2017 as the US's Director of National Intelligence, a position in which he oversaw the activities of 17 distinct government organizations including the National Geospatial-Intelligence Agency, argues strongly in his recent autobiography (Clapper 2019) that the gathering, assembly, and interpretation of intelligence should be driven by a simple vision: the speaking of truth to power. The policy decisions that result from that intelligence are the responsibility 
of other leaders and branches of government to whom the intelligence community (IC) reports, and should not bias or distort the community's primary function. We could argue, then, that the value of urban informatics lies in the scientific quality of the data acquired, and the compilations, interpretations, analyses, and visualizations performed. Urban informatics should be replicable so that independent investigators should reach the same conclusions, should capture and address uncertainties, and should use terms, definitions, and practices that are as far as possible shared and standardized. The urban IC should be driven by an objective of speaking truth to urban power, whether it be city administration, elected representatives, or the urban public.

Is this a useful vision for urban informatics? It is certainly aligned with much writing on smart cities. Its ultimate goal would be the development of data acquisition programs to capture a representation of the city and its enormous complexityas close as possible to a digital twin-that could then support the city's decisionmaking processes. It implies a simple kind of accountability, and a taxonomy of different kinds of intelligence somewhat comparable to the signals intelligence (SIGINT), geospatial intelligence (GEOINT), intelligence derived from social media and other social sources (HUMINT), etc., of the IC. But there are several compelling alternatives.

\subsubsection{Urban Science}

Many chapters, especially those in Part IV, are driven by the traditional goals of science: the acquisition of knowledge about urban systems. Such knowledge should be generalizable, since urban science looks for processes that are replicable across many urban environments. Just as physics searches for general laws and principles, it would be of little interest in urban science to discover knowledge about London, or some part of London, that cannot usefully be applied and implemented in other cities and neighborhoods, at least in those that bear some resemblance to London; and cannot be usefully applied at other times. Urban science is driven by the belief that such general principles exist, and can be discovered through the kinds of natural experiments that rely on observations, public-sector programs that gather statistical data, crowdsourcing, remote sensing, and data that can be cajoled from the private sector's enormous stocks.

Geography as a discipline has long struggled with finding a balance between the search for general principles on the one hand, and the documentation of the unique on the other, since the latter is after all what drove the Age of Discovery in Portugal and the explorations that have always captivated the human imagination. It concerned Varenius, the Polish-Dutch geographer of the seventeenth century (Warntz 1989), who wrote about what he termed Special (idiographic) Geography and General (nomothetic) Geography. It drove a debate in the 1950s between Schaefer and Hartshorne (Harvey 1969) that remains a cornerstone of graduate courses in 
geographic thought. The more prestigious sciences will often describe idiography using perjorative (to them) terms such as "journalism" and "mere description."

Today, this debate has become more nuanced. Techniques such as geographically weighted regression (GWR; Fotheringham et al. 2002) and local indicators of spatial association (LISA; Anselin 1995) represent a form of compromise: a set of structures whose forms can be generalized, but whose parameters are allowed to vary in space and perhaps also in time. We might term this weak generalizability, and several arguments can be presented in its favor. In the social and environmental sciences, it is hard to imagine any principle being truly deterministic, since there will always be unaccounted factors. In short, the goal of an $\mathrm{R}^{2}$ of 1 will always be unattainable. If those unspecified factors vary spatially, then the effect will be a spatial variation in the parameters of the model. Alternatively, we might argue that processes do truly vary with location: that growing up in Detroit is fundamentally different from growing up in New Orleans, all other things being equal.

If urban science is indeed driven by curiosity, then its responsibilities end when knowledge is shared through the process of publication. Application and implementation become the responsibility of others, as in the first vision of urban intelligence, and one can imagine an applied urban science emerging that is devoted to the use of general urban knowledge - or perhaps, it would be better termed urban engineering. The value proposition is now different: instead of the abstract concepts of understanding and explanation that drive curiosity-driven science, applied urban science would be accountable through its broader impacts.

\subsubsection{Urban Planning and Design}

The fifth $\mathrm{V}$ has already taken two different meanings in these sub-sections. Value in the case of urban intelligence will be determined by policy- and decision-makers, based on the degree of support given by the information provided to them. In the case of urban science, value derives in the first instance from the production of generalizable knowledge, and less directly from its usefulness in application. But the urban planning and design that have been discussed in several chapters of this book proceed according to a prior definition of value: the extent to which plans and designs are consistent with agreed principles. In short, they are normative, unlike the previous two visions. In some cases, these principles may be at least partially embedded in software, as in Chap. 35 and in the broader area of spatial optimization, which seeks to design solutions to problems that are optimal against defined objectives.

Many issues complicate that simple vision. First, except in the simplest instances, it will be difficult to reach an agreement on the principles that drive planning and design. Will they serve the interests of a minority at the expense of the majority? Will they adequately address the needs of those whose voices are often muted or unheard? The field of multicriteria decision-making has evolved as a model of how decisions can be made in the face of conflicting goals; its tools include methods for determining consensus weights to be applied to alternative numerical criteria 
(Saaty 1977). Second, while we might argue that a decision based on agreed criteria is inherently more fair, in practice any solution is bound to be seen to favor one position or another.

\subsubsection{Urban Development}

The value proposition for business is of course a matter of simple economics: innovations are driven in the first instance by their ability to make money. While disruptions such as Uber or dockless bikes can certainly have redeeming social value, it is their eventual profitability that ultimately drives their growth. Many businesses invite the users of their apps to allow locations to be shared and may argue that the result will be more specific information to the user. This is the case for wayfinding apps, and also for many news or weather apps. But the business case for such apps relies at least in part on the market value of those user locations to retailers, advertisers, and others. This trading of location data will be consistent with the app's terms and conditions of use, but the user is unlikely to have taken the time to read their tens of pages of fine print and to have realized what they imply.

\subsection{Unintended Consequences}

Although the previous section has outlined how value can be assessed under different visions of urban informatics, it is often the unintended consequences of actions and developments that determine whether outcomes will eventually be assessed positively or negatively. How, for example, should we assess the impacts of online shopping? The individual citizen benefits from having goods delivered quickly, without the time and expense of a shopping trip. New jobs are created in the city's delivery industry, and profits are made by the owners of shopping Web sites and their suppliers. But the impact on traditional shopping is severe, with significant loss of local employment and the closure of conventional retail businesses, and in some cases, wholesale abandonment of shopping centers. Supply chains may have to be reorganized, and the city's function as a regional shopping center may be undermined.

The advent of connected and autonomous vehicles (CAVs) provides a suitable case in point. Already many new vehicles are connected to the Internet, and capable of reporting details of location, driving habits, and even driver biometrics. Such data can be useful to the parents of young drivers, to insurance companies following a crash, and to mechanics when a vehicle is serviced. They have commercial value, as already noted in Sect. 48.2.4. But they also potentially have more sinister value to traffic-control systems and law enforcement, and to what has been termed automated social control (New York Times 2020).

Cities are complex phenomena, performing functions that are not only internal but also regional and global. The growth of IoT will benefit the city through the services 
it provides, but will also benefit employment in high-tech industries in cities that may be half a world away; and the waste created by the city will almost certainly be exported to the city's hinterland, to areas downwind and downstream, and to foreign markets for recycled material. What may be out of sight and out of mind to a city's citizens may be very real to people elsewhere in the world.

\subsection{The Future of Urban Informatics}

Whether as a means for gathering urban intelligence, or as a basis for new urban science, or as a tool for planning and design, or as a source of profit for developers, urban informatics is clearly destined for accelerating growth. There is little danger of it experiencing a quick death as a short-term fad. Yet it can also be a source of the future dystopia, given its potential for surveillance and control.

This short piece has drawn attention to two issues: the different ways in which parts of the urban informatics community address the value of what they are doing; and the temptation to focus on the internal complexity of the city without addressing the complexity of its external linkages.

There are obvious similarities between the emerging field of urban informatics in 2020 and the state of GIS in the early 1990s: both are growing strongly, with enormous promise. It is important therefore that the kinds of concerns for broader social impacts that emerged at that time in the GIS research community, and led to an outpouring of important research, should also become part of the agenda of urban informatics. We are the people to explore these broader impacts and to raise these issues with our governments and with the public.

\section{References}

Anselin L (1995) Local indicators of spatial association-LISA. Geogr Anal 27(2):93-115

Clapper JR (2019) Facts and fears: hard truths from a life in intelligence. Penguin, New York

Fotheringham AS, Brunsdon C, Charlton M (2002) Geographically weighted regression: the analysis of spatially varying relationships. Wiley, Chichester

Harvey D (1969) Explanation in geography. Edward Arnold, London

New York Times (2020) https://www.nytimes.com/2020/03/01/business/china-coronavirus-survei llance.html. Accessed 13 April 2020

Pickles J (ed) (1995) Ground truth: the social implications of geographic information systems. Guilford, New York

Saaty TL (1977) A scaling method for priorities in hierarchical structures. J Math Psychol 15:234281

Schuurman N (2000) Trouble in the heartland: GIS and its critics in the 1990s. Prog Hum Geogr 24(4):569-590

Smith N (1992) History and philosophy of geography: real wars, theory wars. Prog Hum Geogr 16(2):257-271 
Taplin JT (2017) Move fast and break things: how Facebook, Google, and Amazon cornered culture and undermined democracy. Little, Brown, New York

Warntz W (1989) Newton, the newtonians, and the geographia generalis varenii. Ann Assoc Am Geogr 79(2):167-191. https://doi.org/10.1111/j.1467-8306.1989.tb00257.x

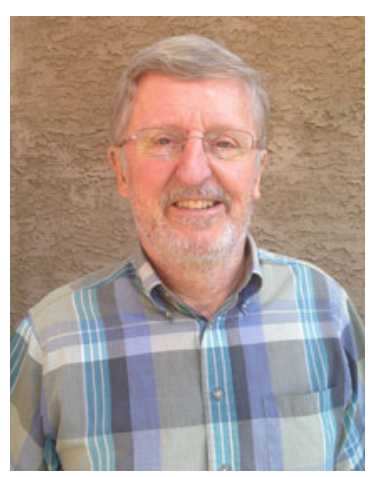

Michael F. Goodchild is Emeritus Professor of Geography at the University of California, Santa Barbara, a Distinguished Chair Professor at The Hong Kong Polytechnic University and a Research Professor at Arizona State University. He is a member of the US National Academy of Sciences, and he is interested in GIScience.

Open Access This chapter is licensed under the terms of the Creative Commons Attribution 4.0 International License ( http://creativecommons.org/licenses/by/4.0/), which permits use, sharing, adaptation, distribution and reproduction in any medium or format, as long as you give appropriate credit to the original author(s) and the source, provide a link to the Creative Commons license and indicate if changes were made.

The images or other third party material in this chapter are included in the chapter's Creative Commons license, unless indicated otherwise in a credit line to the material. If material is not included in the chapter's Creative Commons license and your intended use is not permitted by statutory regulation or exceeds the permitted use, you will need to obtain permission directly from the copyright holder.

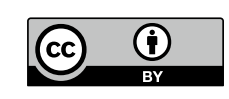

